\title{
Randomised trial of mycophenolate mofetil versus azathioprine for treatment of chronic active Crohn's disease
}

M F Neurath, R Wanitschke, M Peters, F Krummenauer, K-H Meyer zum Büschenfelde, J F Schlaak

\begin{abstract}
Background-Crohn's disease is a chronic inflammatory disease of the alimentary tract. Azathioprine is an effective agent in the management of chronic active Crohn's disease leading to long term remission of disease activity. Such treatment leads to limited efficacy or side effects in a small subset of patients.
\end{abstract}

Aims-To compare efficacy and side effects of treatment with azathioprine plus corticosteroids versus mycophenolate mofetil (MMF) plus corticosteroids in patients with chronic active Crohn's disease.

Methods-Seventy patients with chronic active Crohn's disease (Crohn's disease activity index (CDAI) greater than 150) were randomised for treatment with azathioprine/cortisone or MMF/cortisone. Corticosteroid dosage was tapered according to a standard protocol. Disease activity was monitored by clinical scores after one, two, three, and six months.

Results-Treatment of patients with moderately active (CDAI 150-300) Crohn's disease with $M M F / c o r t i s o n e$ led to a significant reduction in clinical activity scores comparable to treatment with azathioprine/cortisone. Treatment of patients with highly active Crohn's disease (CDAI greater than 300) with MMF/ cortisone caused significant suppression of clinical activity earlier than azathioprine/ cortisone treatment. Treatment with MMF/ cortisone was associated with few adverse effects.

Conclusion-Treatment of chronic active Crohn's disease with MMF plus cortisone appears to be effective and well tolerated and should be considered in patients allergic to azathioprine or in whom azathioprine has failed.

(Gut 1999;44:625-628)

Keywords: mycophenolate mofetil; azathioprine; Crohn's disease

Department of

Medical Statistics,

University of Mainz,

Langenbeckstrasse 1,

55101 Mainz, Germany

F Krummenauer

Correspondence to: Dr Neurath.

Accepted for publication 8 December 1998

Crohn's disease is a chronic inflammatory disease of the gastrointestinal tract of unknown origin. ${ }^{1-3}$ It is characterised by a discontinuous transmural, granulomatous inflammation leading to intestinal fibrosis, strictures, and ileus. ${ }^{23}$ There is growing evidence that Crohn's disease is associated with a dysregulated intestinal immune response of CD4+ $\mathrm{T}$ helper 1 type lymphocytes that produce high amounts of proinflammatory cytokines such as interferon (IFN) $\gamma$ and tumour necrosis factor (TNF) ${ }^{4-6}$ Although treatment with corticosteroids is highly successful at decreasing symptoms in active disease, subgroups of patients treated with these agents continue to have complications of the disease and many patients suffer from steroid toxicity. ${ }^{237}$ Azathioprine combined with corticosteroids has been shown to cause earlier responses compared with monotherapy with corticosteroids and may allow a reduction in steroid dose. ${ }^{7}$ Treatment with azathioprine has been shown to be effective in treating active Crohn's disease (36-76\% response rates at $1.5-3 \mathrm{mg} / \mathrm{kg} /$ day) and in maintaining long term remission of disease activity. ${ }^{78}$ However, a recent meta-analysis showed that azathioprine therapy has an $8.9 \%$ incidence of adverse effects with a pooled odds ratio of 5.26 for development of adverse effects severe enough to require withdrawal of the drug. ${ }^{8}$ Similarly, Present et al reported adverse effects such as significant infection $(7.4 \%)$, pancreatitis $(3.3 \%)$, neoplasm $(3.1 \%)$, bone marrow suppression $(2.0 \%)$, allergy $(2 \%)$, and drug hepatitis $(0.3 \%)$ in some azathioprine treated patients. ${ }^{9}$ Based on these observations, alternative treatment options may be desirable for subgroups of patients with Crohn's disease.

Mycophenolate mofetil (MMF) is an ester prodrug of mycophenolic acid that inhibits inosine monophosphate dehydrogenase and potently suppresses lymphocyte proliferation. ${ }^{10-12}$ It reduces production of IFN- $\gamma$ (but not TNF) by $\mathrm{T}$ cells more potently than does methotrexate. ${ }^{13} 14$ MMF has been successfully used in organ transplantation to prevent graft rejection. ${ }^{15}$ Furthermore, various clinical studies have shown its efficacy in suppressing autoimmune and chronic inflammatory disorders, such as rheumatoid arthritis, ${ }^{16}$ pemphigus vulgaris, ${ }^{17}$ and psoriasis. ${ }^{18}$ Here, we show that combined treatment with MMF plus cortisone appears to be effective and well tolerated in active Crohn's disease.

\section{Methods}

A randomised prospective study was conducted at the I. Department of Medicine of the University of Mainz between November 1996 and November 1997.

Abbreviations used in this paper: CDAI, Crohn's disease activity index; MMF, mycophenolate mofetil; IFN, interferon; TNF, tumour necrosis factor. 


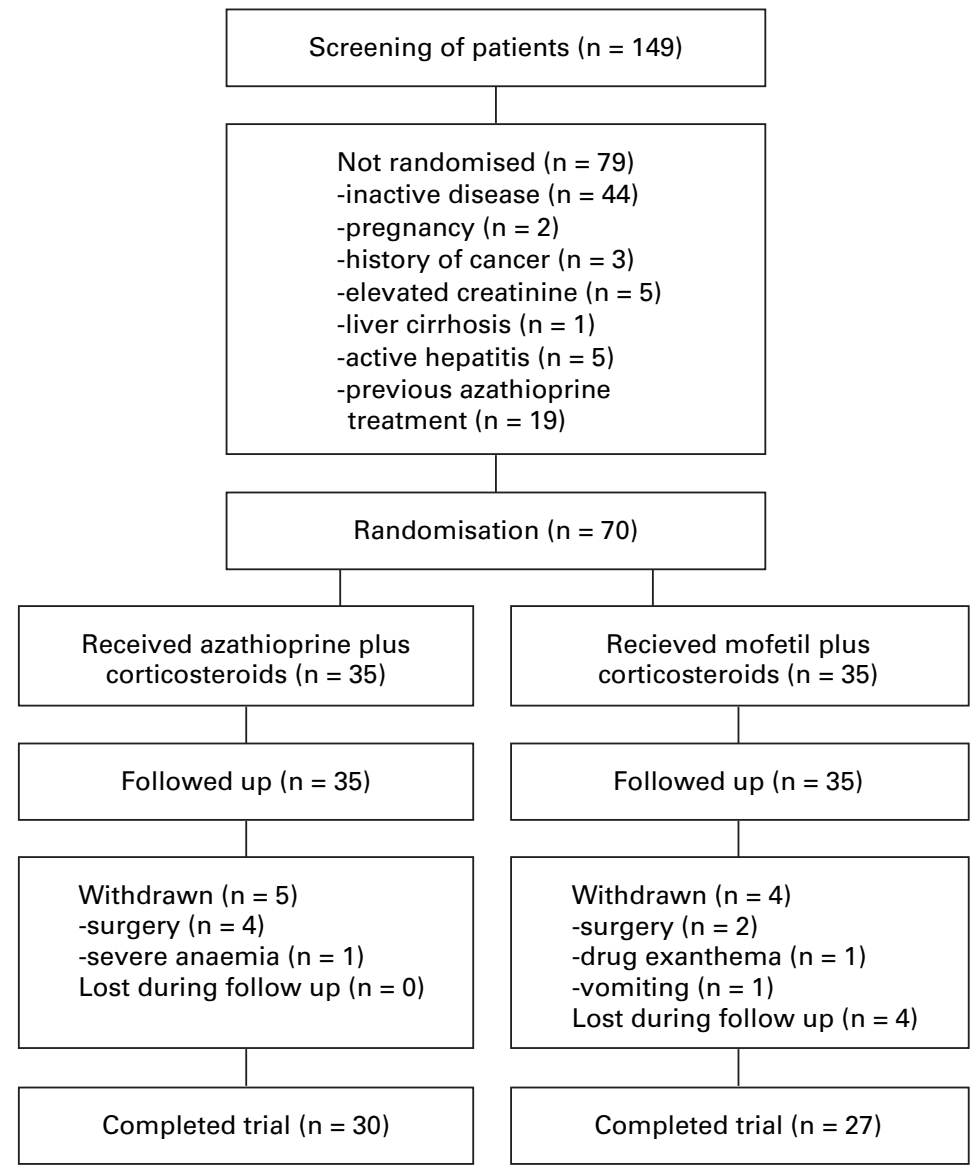

Figure 1 Progress of patients during the randomised trial. ${ }^{27}$

PATIENTS

Eligible patients had chronic active Crohn's disease for at least one year and had a minimum of three acute flares within the previous three years. The Crohn's disease activity index (CDAI) was higher than 150 points in all patients. Patients with CDAI values below 150 were considered to have inactive disease and were not eligible. Before inclusion patients underwent endoscopic (colonoscopy) and radiological (barium contrast studies) evaluation; patients included in the study showed active mucosal inflammation. Patients without active mucosal inflammation

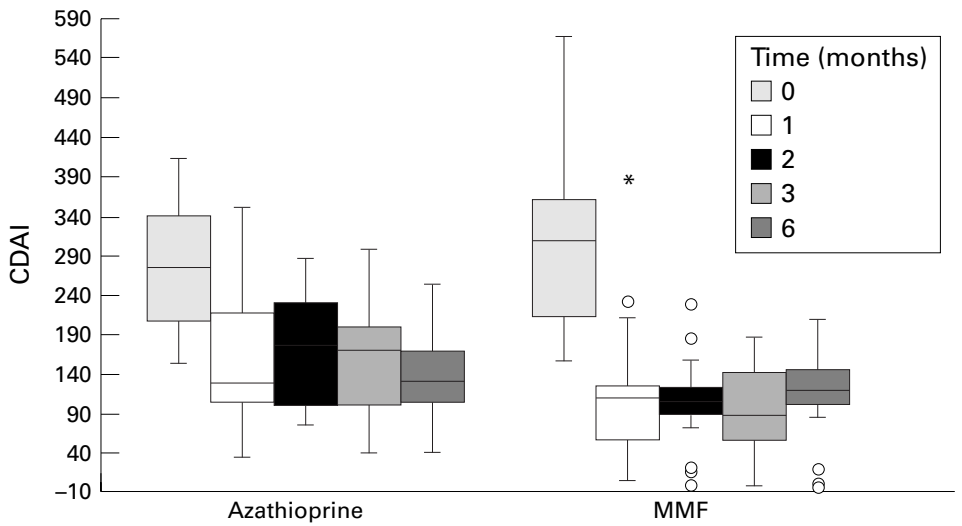

Figure 2 CDAI scores of all Crohn's patients before and after treatment with MMF or azathioprine. CDAI scores were determined at indicated time points and are shown in Box/Whisker plots. Points (circles and asterisks) outside the plots represent values outside the 2 (3) SD interval.
Table 1 Group comparison by median changes in CDAI at indicated time points compared with the previous $C D A I$ score

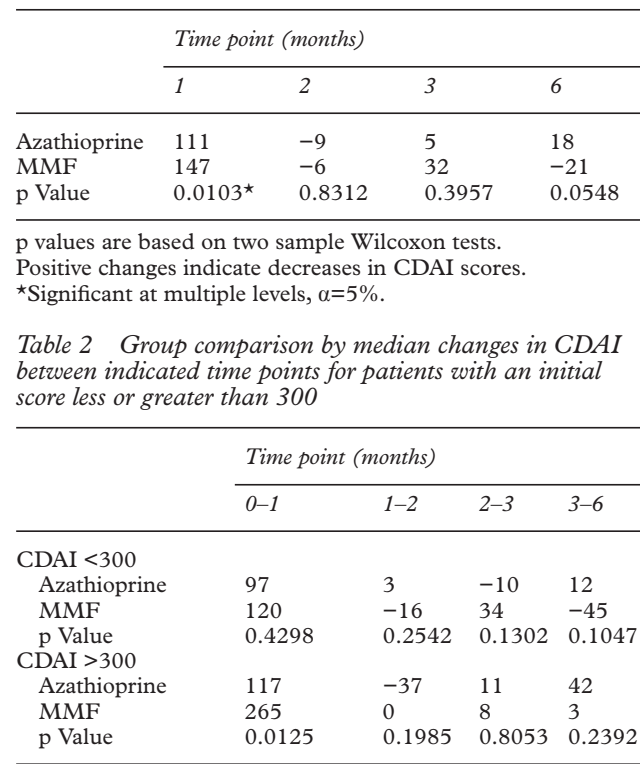

$\mathrm{p}$ values are based on two sample Wilcoxon tests.

Positive changes indicate decreases in CDAI scores.

were excluded. Further exclusion criteria were pregnancy, elevated creatinine, history of cancer, liver cirrhosis, chronic active hepatitis, and previous treatment with azathioprine or MMF.

RANDOMISATION

Seventy patients with CDAI scores greater than $300(n=35)$ or less than $300(n=35)$ were assigned by random numbers, in a $1: 1$ ratio, to receive $2.5 \mathrm{mg} / \mathrm{kg}$ azathioprine plus $50 \mathrm{mg}$ prednisolone or $15 \mathrm{mg} / \mathrm{kg} \mathrm{MMF}$ plus $50 \mathrm{mg}$ prednisolone orally. It should be noted, however, that neither patients nor the investigators were blinded to the randomisation sequence and the patients knew which medication they were on.

PREDNISOLONE TREATMENT

Corticosteroid dosage was decreased each week according to the following regimen: 50 $\mathrm{mg}, 40 \mathrm{mg}, 30 \mathrm{mg}, 25 \mathrm{mg}, 20 \mathrm{mg}, 15 \mathrm{mg}, 10$ $\mathrm{mg}$, and $5 \mathrm{mg}$ per day. Prednisolone $5 \mathrm{mg}$ was then given as maintenance therapy during the six month observation period. Patients whose condition worsened had their prednisolone dosage increased to a dose of $40 \mathrm{mg}$ per day. The tapering regimen described above was then started again.

FOLLOW UP

Patients were seen one, two, three, and six months after randomisation. At each visit, the CDAI score was determined. Patients collected the diary data for calculation of the CDAI in the week prior to their clinic visit. Serum concentrations of aminotransferases, alkaline phosphatase, red and white blood cells, lipase, amylase, and creatinine were also determined.

\section{STATISTICAL ANALYSIS}

The confirmatory hypothesis of different CDAI profiles under azathioprine versus MMF treatment was tested via pairwise two sample 


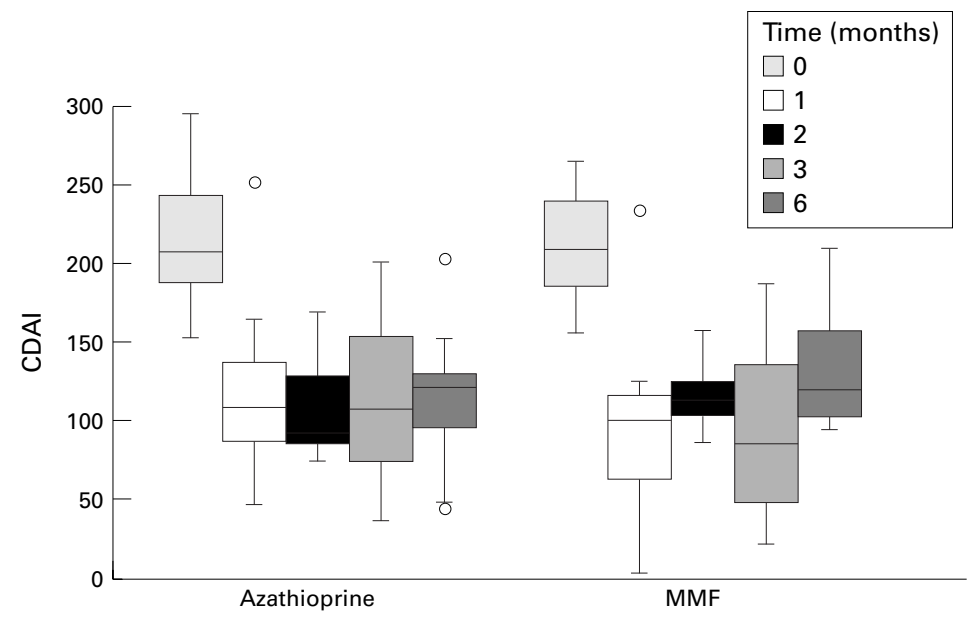

Figure 3 CDAI scores of Crohn's patients with initially moderate activity (CDAI 150-300). CDAI scores were determined at indicated time points and are shown in Box/Whisker plots. Points (circles) outside the plots represent values outside the 2 (3) SD interval.

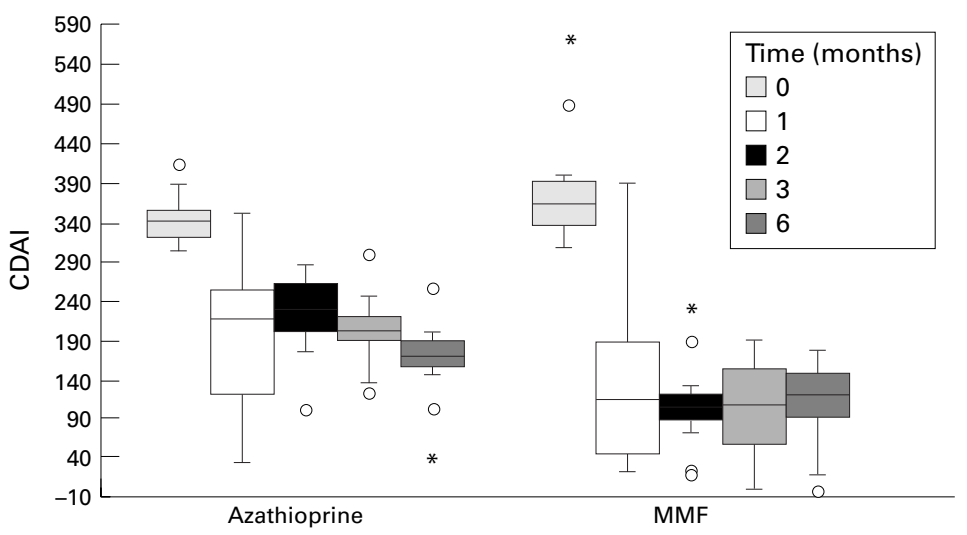

Figure $4 C D A I$ scores of Crohn's patients with initially high activity $(C D A I>300)$. $C D A I$ scores were determined at indicated time points and are shown in Box/Whisker plots. Points (asterisks and circles) outside the plots represent values outside the 2 (3) SD interval. AZA, azathioprine. were performed with SAS and SPSS for Windows packages.

\section{Results}

Between November 1996 and May 1997, 149 patients were screened for the study, 79 of whom were excluded (fig 1). Seventy patients with chronic active Crohn's disease as defined by the criteria specified in Methods were selected for the study. Patients were randomly assigned to receive $\mathrm{MMF}$ or azathioprine plus corticosteroids. Baseline characteristics of the patients such as location of disease (ileum: $n=14$ versus $n=12$; ileum and colon: $n=12$ versus $n=13$; and colon: $n=9$ versus $n=10$, respectively) were not significantly different between the treatment groups. Four patients in the MMF group were lost during follow up.

Figure 2 and table 1 show treatment outcome based on the CDAI. In both groups the percentage of patients who fell 75-100 points in the CDAI during the first month was relatively high (74\% in the azathioprine versus $85 \%$ in the MMF group), possibly due to the concomitant corticosteroid treatment. In patients with moderate activity (CDAI 150-300), the average CDAI scores decreased in both treatment groups, with scores dropping to approximately those of patients with inactive disease (CDAI less than 150) after one month of treatment (fig 3). In these patients the mean scores between the two patient groups were not statistically different over the entire six month observation period. In contrast, in patients with highly active Crohn's disease (CDAI more than 300) the group treated with MMF had a significantly greater decrease in the CDAI score during the first month of treatment (fig 4; table 2). This was associated with a greater percentage of patients entering remission in the MMF group (fig 5). Between two and six months the CDAI scores in MMF treated patients remained stable at a comparable level for both subgroups $(p=0.7012$ for Wilk's lambda). In contrast, in the azathioprine treated group with high initial activity (CDAI greater than 300) there was a clear trend for a continuous decrease in the CDAI scores over the six month observation period $(\mathrm{p}<0.001$ for Wilk's lambda). Finally, in the azathioprine treated group of patients with highly active

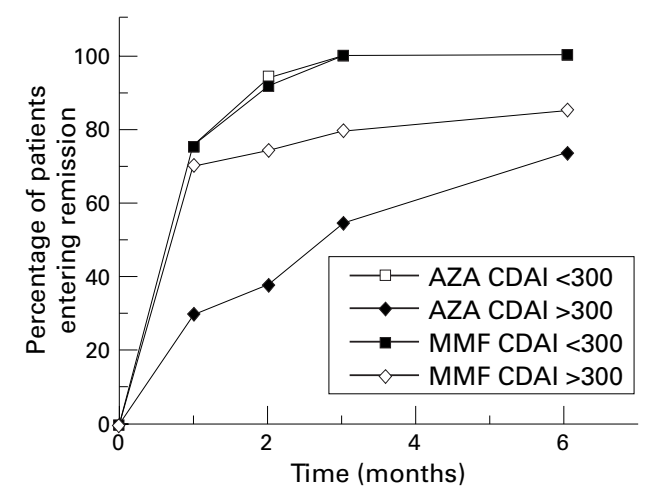

Figure 5 Cumulative percentage of patients entering remission at indicated time points during the six month observation period. 
disease, four patients had to undergo surgery due to strictures and ileus, compared with two patients in the MMF group.

Treatment with both azathioprine/cortisone and $\mathrm{MMF} /$ cortisone appeared to be well tolerated. Among all patients treated with azathioprine, seven showed adverse events such as nausea $(n=4)$, transient cholestasis $(n=2)$, and anaemia $(n=1)$ compared with two patients in the MMF treated group who developed drug exanthema and vomiting, respectively.

\section{Discussion}

We found that MMF combined with prednisolone seems to be an effective and well tolerated treatment for patients with active Crohn's disease. Thus, in patients with moderately active Crohn's disease (CDAI 150-300) MMF treatment was as effective as azathioprine treatment (both in combination with steroids) at reducing CDAI scores to levels seen in clinical remission (CDAI less than 150). Furthermore, in patients with highly active disease (CDAI greater than 300) treatment with MMF plus prednisolone led to an earlier reduction in the CDAI score which contrasted with the relatively slow onset of therapeutic effects obtained with the antimetabolite azathioprine in this and in previous studies. ${ }^{19-21}$ As low dose steroid treatment was maintained over the six month observation period in both groups in this study, we propose to determine the requirement for concomitant steroid therapy for MMF effects in consecutive trials. Finally, we observed that treatment of Crohn's disease with MMF appears to be well tolerated in our group of patients. In this regard, surprisingly, no cases of diarrhoea occurred on MMF treatment, in contrast to previous studies using MMF for treatment of kidney transplant recipients. ${ }^{115}$ This was possibly due to the lower dosage of the drug that was used in this study (1.5 versus $2-3$ $\mathrm{g} /$ day) or due to the lack of other additional immunosuppressive drugs such as antilymphocyte globulin or cyclosporin A.

Effective drug therapy to induce and maintain clinical remission in patients with Crohn's disease is currently a major clinical problem. Maintenance therapy with aminosalicylates, prednisolone, budesonide, methotrexate, and azathioprine has been previously tested. ${ }^{30-24}$ Furthermore, recombinant interleukin 10 and antibodies to TNF have been recently suggested to be a therapeutic alternative for patients with moderate to severe disease activity. ${ }^{25}{ }^{26}$ In the present study, we found that treatment with mofetil plus prednisolone appeared to be effective in inducing remission in patients with active Crohn's disease, whereas a recent preliminary study in eight patients showed that MMF had limited efficacy in maintaining remission. No severe adverse effects were noted. Thus, in our group of patients, immunosuppression with $\mathrm{MMF} /$ cortisone was well tolerated in active Crohn's disease. Such treatment may therefore be con- sidered in patients allergic to azathioprine or who have failed to respond to azathioprine. We propose that the therapeutic efficacy of MMF in chronic active Crohn's disease should be confirmed by prospective randomised double blind studies.

The authors gratefully acknowledge Warren Strober (National Institute of Health, NIAID) for critical reading of the manuscript.

1 Strober W, Fuss I, Kelsall B, et al. Mucosal immune regulation and dysregulation: the pathogenesis of inflammatory bowel disease. In: Sleisinger MH, ed. Gastrointestinal disease. Pathophysiology, diagnosis, management. Update 4. disease. Pathophysiology, diagn

2 Hanauer SB. Inflammatory bowel disease. $N$ Engl $7 \mathrm{Med}$ 1996;334:841-8.

3 Kornbluth A, Salomon P, Sachar DB. Crohn's disease. In: Sleisinger MH, ed. Gastrointestinal disease. Pathophysiology, diagnosis, management. Update 4. WB Saunders, 1996: 1270-304.

4 Neurath MF, Fuss I, Kelsall BL, et al. Antibodies to IL-12 abrogate established experimental colitis in mice. $\mathcal{f}$ Exp Med 1995;182:1280-9

5 Fuss I, Neurath MF, Boirivant M, et al. Disparate CD4+ lamina propria (LP) lymphocyte secretion profiles in inflammatory bowel disease. F Immunol 1996;157:1261-70.

6 Breese E, MacDonald TT. TNF-alpha secreting cells in normal and diseased human intestine. Adv Exp Med Biol 1995;371B:821-4

7 Ewe K, Press AG, Singe CC, et al. Azathioprine combined with prednisolone or monotherapy with prednisolone in active Crohn's disease. Gastroenterology 1993;105:367-72.

8 Pearson DC, May GR, Fick GH, Sutherland LR. Azathioprine and 6-mercaptopurine in Crohn's disease. A meta-analysis. Ann Intern Med 1995;122:132-42.

9 Present DH, Meltzer SJ, Krumholz MP, et al. 6 mercaptopurine in the management of inflammatory bowel disease: hort- and long-term toxicity. Ann Intern Med 1989;111: 641-9.

0 Lipsky JJ. Mycophenolate mofetil. Lancet 1996;348:1357-9.

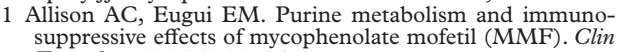
Transplant 1996;10:77-84.

12 Allison AC, Eugui EM. Immunosuppressive and other effects of mycophenolic acid and an ester prodrug, mycophenolate mofetil. Immunol Rev 1993;136:5-28.

13 Hildner K, Märker-Hermann E, Schlaak JF, et al. Azathioprine and mycophenolate mofetil specifically modulate cytokine production by murine T cells. Ann N Y Acad Sci (in press).

14 Neurath MF, Hildner K, Becker C, et al. Methotrexate specifically modulates cytokine production by $\mathrm{T}$ cells and macrophages in collagen-induced arthritis. A mechanism for methotrexate-mediated immunosuppression. Clin Exp Immunol (in press)

15 European Mycophenolate Mofetil Cooperative Study Group. Placebo-controlled study of mycophenolate mofetil combined with cyclosporin and corticosteroids for prevention of acute rejection. Lancet 1995;345:1321-5.

16 Goldblum R. Therapy of rheumatoid arthritis with mycophenolate mofetil. Clin Exp Rheumatol 1993;11:S117-19.

17 Enk AH, Knop J. Treatment of pemphigus vulgaris with mycophenolate mofetil. Lancet 1997;350:494.

18 Epinette WW, Parker CM, Jones EL, et al. Mycophenolic acid for psoriasis. I Am Acad Dermatol 1987;17:962-71.

19 Present DH, Korelitz BI, Wisch N, et al. Treatment of Present $\mathrm{DH}$, Korelitz $\mathrm{BI}$, Wisch $\mathrm{N}$, et al. Treatment of
Crohn's disease with 6-mercaptopurine. $N$ Engl $\mathcal{f}$ Med 1980;302:981-7.

20 Rhodes J, Bainton D, Beck P, et al. Controlled trial of azathioprine in Crohn's disease. Lancet 1971;ii:1273-6.

21 O'Donoghue DP, Dawson AM, Powell-Tuck J, et al. Double-blind withdrawal trial of azathioprine as maintenance treatment for Crohn's disease. Lancet 1978;ii:955-7.

22 Bouhnik Y, Lemann M, Mary JY, et al. Long-term follow-up of patients with Crohn's disease treated with azathioprine or 6-mercaptopurine. Lancet 1996;347:215-19.

23 Sachar DB. Budesonide for inflammatory bowel disease: is it a magic bullet? N Engl f Med 1994;331:873-4.

24 Feagan BG, Rochon J, Fedorak RN, et al. Methotrexate for the treatment of Crohn's disease. N Engl F Med 1995;332: 292-7.

25 Targan SR, Hanauer SB, van Deventer SJH, et al. A short-term study of chimeric monoclonal antibody cA2 to tumor necrosis factor alpha for Crohn's disease. $N$ Engl $\mathcal{F}$ Med 1997;337:1029 al

26 Van Deventer SJ, Elson CO, Fedorak RN. Multiple doses of intravenous interleukin-10 in steroid-refractory Crohn's intravenous interleukin-10 in steroid-ref
disease. Gastroenterology 1997;113:383-9.

27 Farthing MJG, Newcombe RG. Reporting of clinical trials in Gut: the CONSORT statement. Gut 1997;40:563-4.

28 Fellermann K, Steffen M, Stein J, et al. Mycophenolate mofetil in chronic active inflammatory bowel disease-a preliminary report. FALK Symposia 1998;105:A40 